\title{
Positive contrast obtained in rats following a shift in schedule, delay, and magnitude of reward
}

\author{
MITRI E. SHANAB and GERALD CAVALLARO \\ California State University, Fresno, California 93719
}

\begin{abstract}
Sixty-four rats were used in a 2 by 2 by 2 factorial design (Phase 1) which combined two levels of delay schedule (partial vs. continuous), two levels of magnitude of reward (1 vs. 22 pellets), and two levels of delay of reward (10 vs. $30 \mathrm{sec})$. On each trial in Phase 2, each subject received 22 pellets after a 10-sec delay interval. A significant positive contrast effect was obtained when all three variables of magnitude, delay, and schedule were upshifted. Similarly, positive contrast was obtained based on a shift in delay and schedule, magnitude and schedule, and magnitude and delay. Moreover, positive contrast was obtained based on either an upward shift in schedule or magnitude. A simple decrease in delay of reward did not produce positive contrast. The results were viewed as favoring relative rather than absolute theories of reinforcement. However, a modified sequential (capaldian) interpretation of positive contrast was also offered.
\end{abstract}

Recent reviews of the runway literature cite an impressive number of studies that have consistently reported an absence of a positive contrast effect (PCE), i.e., subjects shifted from a small to a larger reward apparently do not exceed the performance level of control subjects receiving the larger reward all the time (Black, 1968; Dunham, 1968). While such a finding is in agreement with an absolute theory of reinforcement (Capaldi, 1967), it runs counter to other reinforcement theories that emphasize the relative effectiveness of reinforcers (Helson, 1964). Proponents of the relative view have suggested that the lack of a PCE in the runway could be attributed to a ceiling effect inherent in the running response (Bower, 1961). The ceiling effect hypothesis has since received considerable support (Mellgren, 1972; Shanab, Birnbaum, \& Cavallaro, 1974; Shanab, Sanders, \& Premack, 1969). Shanab et al. (1974) discuss several of the more recent control procedures which have resulted in significant positive contrast in the runway. These procedures were designed to reduce the running speed of the subjects to a submaximal level so as to allow for any positive contrast effects to emerge when an upward shift in reward magnitude was introduced. Thus delay of reward, specially constructed mazes that prevented subjects from running at top speed, punishment, and reduced drive level have been effectively used to control for ceiling effects. When upward shifts in reward magnitude were made under these conditions, positive contrast was obtained.

Recently Shanab and Biller (1972) used a new

The research for this paper was supported in part by faculty research Grant 3025.07 awarded to the first author. Reprint requests should be sent to Mitri E. Shanab, Department of Psychology, California State University, Fresno, California 93740.

The second author is now at Carleton University, Ottowa, Canada. procedure to obtain a PCE in the runway. Not only was delay used to control for ceiling effects, but the shift was based on a large incentive difference. Specifically, different groups of subjects received initially either small or large reward following a delay of either 0,15 , or $30 \mathrm{sec}$. When all subjects were shifted to large reward received after $15 \mathrm{sec}$, only the double shift, i.e., those subjects that were shifted from a small reward received after long delay $(30 \mathrm{sec})$ to a large reward received after $15 \mathrm{sec}$, showed positive contrast. No PCE was obtained on the basis of single shifts, i.e., a simple upward shift in magnitude or simple decrease in delay of reward. It should be noted that Marx (1969), using the Skinner box obtained a PCE based on a large incentive difference which involved a qualitative rather than a quantitative shift in reinforcement.

These findings (Marx, 1969; Shanab et al., 1972) seem to suggest that the ceiling effect does not necessarily have to represent a physiological limit only but could also represent a psychological limit. In other words, to show a PCE, the organism may require a greater amount of incentive motivation than is usually provided by the conventional single upward shift in reinforcement conditions. This would explain why the double shift in reinforcement conditions in the Shanab et al. study (1972) was more effective than the single shift. Such reasoning leads to the general hypothesis that the greater the incentive difference during the shift phase the more likely that a PCE would be obtained. The present study was designed to test this hypothesis by employing a design that involved three single shifts, three double shifts, and most importantly, a triple shift. Since delay was used throughout to control for ceiling effects, it was expected that all shifts would lead to positive contrast effects and that by implication the PCE would be an increasing function of the incentive differences between pre- and postshift reinforcement conditions. 


\section{METHOD}

\section{Design}

The study consisted of two phases. In Phase 1 a 2 by 2 by 2 factorial design was used, combining two levels of magnitude of reward (1 and $2245-\mathrm{mg}$ food pellets), two levels of delay (10 and $30 \mathrm{sec}$ ), and two levels of schedule (partial and continuous). This resulted in the following eight groups: 1-30-P, 1-30-C, 1-10-P, 1-10-C, 22-30-P, 22-30-C, 22-10-P, and 22-10-C (the first two numbers stand respectively for the number of pellets and duration of the delay interval, whereas the letters $P$ and $C$ stand for partial and continuous delay of reinforcement schedules). The partial delay of reinforcement schedule consisted of $50 \%$ delayed trials and 50\% nonrewarded trials, whereas all trials were delayed in the continuous delay of reinforcement schedule. Following each trial in Phase 2 each subject received 22 pellets after a 10 -sec delay interval.

\section{Subjects}

The subjects were 64 male albino rats of the Sprague-Dawley strain, approximately 90 days old at the beginning of the experiment.

\begin{abstract}
Apparatus
A $1.5-\mathrm{m}$ runway made of unpainted redwood was used. The runway was covered with Plexiglas and was $23 \mathrm{~cm}$ high and $10 \mathrm{~cm}$ wide throughout. The startbox was $18 \mathrm{~cm}$ long and $17 \mathrm{~cm}$ wide. The goalbox was L-shaped. The initial section of the goalbox was $30.5 \mathrm{~cm}$ long and $15 \mathrm{~cm}$ wide. At a right angle to this section was a $16 \times 16 \mathrm{~cm}$ section in which the foodcup was placed. The start- and goalboxes were each separated from the run section by guillotine doors. Four sets of photocells were installed in the runway. Interruption of any of the four photobeams started and/or stopped any one of the three Standard Electric timers which measured start, run and goal times. The first, second, third, and fourth photocells were located $6.4,21.5,103$, and $115 \mathrm{~cm}$ from the startbox, respectively. The start, run, and goal times were summed to obtain total time.
\end{abstract}

\section{Procedure}

Upon arrival from the supplier, the subjects were placed on free feeding for 16 days, following which they were placed on a restricted daily ration of $12 \mathrm{~g}$ of Purina Lab Chow. Proper adjustments were made for food eaten in the apparatus to equate drive level. Water was available at all times in the subject's home cage. During the second week of deprivation each subject was handled and allowed to eat a few Noyes pellets from a $10-\mathrm{cm}$ diam glass cup later used in the goalbox. This procedure was followed for 10 days. Following this period each subject was allowed to explore the unbaited apparatus for 2 min per day for 2 days with all circuitry turned off. To adapt the subject to the various noises of the relays and clocks, all circuitry was then turned on and each subject received an additional 4 days of exploration for $2 \mathrm{~min}$ per day. Following this exploration period Phase 1 began which lasted for 48 trials. One trial a day was given to each subject during this phase as well as the succeeding phase.

The subjects were divided into eight main groups that resulted from the factorial combination of two levels each of the reinforcement magnitude, delay, and delay schedule factors. The reinforcement magnitude was either a single $45-\mathrm{mg}$ pellet or 22 such pellets. Half the subjects received their reward after a 10 -sec delay while the other half after a $30-\mathrm{sec}$ delay. For half the subjects, the reward was delayed on $50 \%$ of the trials (the other $50 \%$ of the trials being nonrewarded). The other half of the subjects were continuously reinforced, but the reward was delayed on $100 \%$ of the trials. The sequence of delayed and nonrewarded trials was quasirandorn, subject to the restriction that no more than two delayed or nonrewarded trials occurred consecutively.
Since all subjects in Phase 1 were either delayed or nonrewarded on a certain percentage of their trials, each subject ran to an empty goalbox on all trials. On delayed trials, the baited cup was lowered into the terminal section of the goalbox following the appropriate delay interval. The subject was removed from the goalbox immediately after consumption of the reinforcer. On nonrewarded trials the subject was removed from the goalbox $30 \mathrm{sec} \cdot$ after interrupting the last photobeam. On all trials, as soon as the subject broke the last photobeam, the guillotine door was lowered to prevent retracing.

Following each trial in Phase 2 all subjects were given 22 pellets after a $10-\mathrm{sec}$ delay for a total of 33 trials.

The procedure in Phase 2 provides seven tests of positive contrast. It allows for a test of positive contrast based on single shifts in either reinforcement magnitude (1-10-C vs. 22-10-C), in schedule $(22-10-\mathrm{P}$ vs. $22-10-\mathrm{C})$, or in delay interval (22-30-C vs. $22-10-\mathrm{C})$. Three other tests can be made based on a two-fold shift in either magnitude and schedule (1-10-P vs. $22-10-C)$, in magnitude and delay $(1-30-\mathrm{C}$ vs. $22-10-\mathrm{C})$, and in delay and schedule (22-30-P vs. 22-10-C). Finally, a test of positive contrast can be made based on a three-fold shift that involves all of the variables (1-30-P vs. $22-10-\mathrm{C})$.

\section{RESULTS}

All times were converted into their reciprocals to obtain speed scores. Since the results of the separate analyses of variance performed on the start, run, goal, and total speed measures were essentially the same, only the results of the total speed analysis will be reported.

\section{Phase 1}

A three-way analysis of variance with repeated measures was performed on the mean speed over the last four trials of the preshift phase, yielding significant magnitude and delay effects $[\mathrm{Fs}(1,56)=20.10$ and 14.82 ; both ps <.01] for magnitude and delay, respectively. The schedule effect was not significant, $F<1$. None of the interactions of the main effects was significant. The trials effect was also not significant $[F(3,168)=2.03, p>.05]$. All other interactions with trials were also not significant, indicating that all groups had reached a stable level by the end of Phase 1 . Individual comparisons using the Newman-Keuls test showed that while the main control group, 22-10-C, was not significantly different from Group 22-10-P, it ran significantly faster than all other groups. There was similarly no significant difference between the three partial groups 22-30-P, 1-30-P, and 1-10-P and their respective control groups, 22-30-C, 1-30-C, and 1-10-C (all ps > .05).

\section{Phase 2}

Figure 1 shows that, following the shift in reinforcement conditions, the 1-pellet groups increased their speeds at a faster rate than the 22-pellet groups, but in neither condition was the overshooting abrupt. Separate analyses of variance were performed on the mean total speeds over Blocks 3-8 as well as Blocks 4-8, yielding similar results. Therefore only the analysis over the last five blocks (i.e., 20 trials) will be presented. An analysis of variance with repeated measures was 


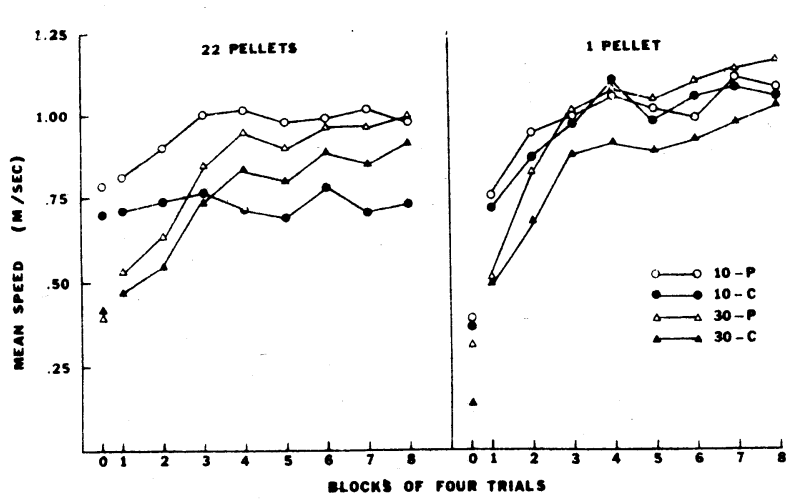

Figure 1. Mean running speed during the shift phase.

performed on the mean speeds over the last five blocks of the shift phase yielding significant magnitude and schedule effects $[\operatorname{Fs}(1,56)=11.86$ and 7.89 , both ps $<.01]$ for magnitude and schedule, respectively. The delay effect was not significant, $F<1$. None of the ensuing interactions was significant. The blocks effect was highly significant $[F(4,224)=4.82, \mathrm{p}<.01]$, indicating, as Figure 1 shows that the running speed of most groups was still increasing. None of the interactions with blocks was significant, showing that the continued improvement in speed was not dependent on the particular preshift condition.

Individual comparisons using the Dunnett test for multiple comparisons with a common control group yielded several interesting results. The triple shift which involved an upward shift in magnitude, delay, and schedule (1-30-P vs. $22-10-\mathrm{C})$ was highly significant $(\mathrm{t}=$ $4.15, \mathrm{p}<.01)$. The three double shifts yielded significant results: A PCE was obtained when an upward shift was made in both magnitude and schedule (1-10-P vs. $22-10-\mathrm{C})(\mathrm{t}=3.61, \mathrm{p}<.01)$, as well as when the upward shift involved increased magnitude and decreased delay $(1-30-\mathrm{C}$ vs. $22-10-\mathrm{C}),(\mathrm{t}=2.49, \mathrm{p}<.05)$. Moreover, the difference between Groups 22-30-P and 22-10-C which reflects an upward shift involving an increase in schedule and decrease in delay was also significant, $(\mathrm{t}=2.52, \mathrm{p}<.05)$. Two of three single shifts yielded significant results. Positive contrast was found both when subjects were shifted from a $50 \%$ to $100 \%$ schedule (22-10-P vs. $22-10-\mathrm{C}),(\mathrm{t}=2.95, \mathrm{p}<.05)$, and from one to 22 pellets $(1-10-\mathrm{C}$ vs. $22-10-\mathrm{C}),(\mathrm{t}=3.62$, $\mathrm{p}<.01)$. However, subjects $(22-30-\mathrm{C})$ shifted from a $30-\mathrm{sec}$ delay to a 10-sec delay did not significantly overshoot the level of their controls $(22-10-\mathrm{C}),(\mathrm{t}=1.52$, $\mathrm{p}>.05$ ).

A simple three-way analysis of variance was performed on the mean speeds over Block 8 in order to determine whether or not the positive contrast effects just reported were still present on the last block of the shift phase. Both the magnitude and schedule main effects were significant $[\mathrm{Fs}(1,56)=12.80$ and 6.49 , both ps $<.01]$, respectively. The delay factor was not significant $[F(1,56)=1.72]$. None of the two-way interactions of the main factors was signficant, Fs $<1$. Moreover, the triple interaction was also not signficant $[F(1,56)=1.89, \mathrm{p}>.05]$. Individual comparisons using the Dunnett test yielded the same results as those reported for the analysis of the last five blocks of Phase 2. Srecifically, the triple shift results (1-30-P vs. 22-10-C) were significant $(\mathrm{t}=4.41, \mathrm{p}<.01)$, as were the results of each of the three double shifts: ts $=3.67$ and 3.06 , both ps $<.01$ for the $1-10-\mathrm{P}$ vs. $22-10-\mathrm{C}$ and the 1-30-C vs. 22-10-C comparisons, respectively, and $\mathrm{t}=$ $2.80, \mathrm{p}<.05$ for the $22-30-\mathrm{P}$ vs. $22-10-\mathrm{C}$ comparison. Similarly, only two of the three single shifts yielded significant results. Both the shifts in schedule (22-10-P vs. $22-10-\mathrm{C})$ and magnitude $(1-10-\mathrm{C}$ vs. $22-10-\mathrm{C})$ were significant $(\mathrm{t}=2.58, \mathrm{p}<.05$, and $\mathrm{t}=3.34, \mathrm{p}<.01$, respectively). The shift in delay (22-30-C vs. 22-10-C) was not significant $(t=1.95, p>.05)$.

Inspection of Figure 1 reveals that the PCE on Block 8, as well as on Blocks 3-8, was an increasing function of the amount of incentive shift. For example, the mean speeds on Block 8 for the nonshifted (control), single shift, double shift, and triple shift subjects were: $.72, .99,1.05$, and $1.17 \mathrm{~m} / \mathrm{sec}$, respectively. It is of interest that the subjects that experienced a double shift in the Shanab et al. study (1972) also had a mean of $1.05 \mathrm{~m} / \mathrm{sec}$. The performance of all eight groups on the last block of the shift phase was subjected to a trend analysis test yielding a highly significant linear component $[\mathrm{F}(1,56=19.37, \mathrm{p}<.001]$.

\section{DISCUSSION}

The results of the present study lend considerable support to the initial hypothesis that upward shifts in reinforcement conditions would result in positive contrast as long as an appropriate control procedure for the ceiling effects is followed. In the present case delay was used to control for ceiling effects and all shifts were superimposed on the delay condition. Of the seven tests for positive contrast which the present study performed, six were statistically significant. Specifically, the triple shift that involved a simultaneous change in magnitude, delay, and schedule produced significant positive contrast, so did the other three double shifts that involves a simultaneous upshift in two of the three variables at a time. Two single shifts, one based on an upward shift in schedule and the other on magnitude resulted in significant positive contrast. The upward shift based on decreased delay did not produce significant positive contrast. The latter finding is in agreement with that obtained by Shanab and Biller (1972) in which a shift from a $30-\mathrm{sec}$ to $15-\mathrm{sec}$ delay produced no positive contrast. The significant positive contrast effect obtained in this study as a result of a shift in both magnitude and delay lend support to the similar finding reported by Shanab et al. (1972). Moreover, the finding of significant double shifts as well as a triple shift in this study supports and extends the results obtained by Shanab et al. (1972). However, the finding of a significant positive contrast effect based on an upward shift in reward magnitude is not in agreement with that found by Shanab et al. (1972). In the latter study the shift was made from 1 to 12 pellets received after a 15 -sec delay, whereas in this study the shift was made from 1 to 22 pellets received after a 10 -sec delay. Perhaps the discrepancy in results could be attributed to the fact that the shift in this study involved a greater incentive difference than the previous study. That a PCE was obtained based on an upward shift in schedule is in agreement with several studies (Harris, Smith \& 
Weinstock, 1962: Leung \& Jensen, 1968; Shanab et al., 1974). It should be noted that the schedule of partial delay used in this study was identical to the traditional partial reinforcement schedule. In other words, as in the traditional case, the $50 \%$ schedule meant that half the trials were randomly rewarded while the other half were not rewarded except that in this case delay was imposed on rewarded trials. This procedure is to be contrasted with the more common partial delay schedule according to which each trial is rewarded, but on a random half of the trials the reward is given immediately while on the other half it is delayed. The continuous schedule in both the traditional and the present procedure is the same: Reward is presented but delayed on each trial. It can thus be seen that while according to the traditional procedure a shift from a partial to a continuous delay schedule constitutes a shift from more favorable to less favorable conditions, by using the present procedure, such a shift involves a change from less favorable to more favorable conditions. This would explain why the schedule effect was independent of the magnitude and delay effects.

The significant linear trend obtained in the shift phase lends strong support to the derivative hypothesis that the PCE is an increasing function of the incentive difference between pre- and postshift. This finding not only supports earlier reports of PCE but carries the interesting implication that the PCE is not an all-or-none phenomenon as probably would be inferred from the available contrast findings. Rather it seems that the PCE is incremental in nature. A graded PCE such as was obtained in this study provides obvious support to any theory that emphasizes the relative rather than the absolute effects of reinforcement. However, an attempt will be made here to explain this and other contrast effects in absolute or associative terms. It should be noted that within the Hullian theoretical framework, both Amsel (1958) and Capaldi (1967) have provided adequate explanations of negative contrast. While Amsel attributed the phenomenon to the detrimental effects of frustration, Capaldi interpreted it in terms of a decrease in habit strength due to generalization decrement as well as a decrease in stimulus intensity due to a change from a larger to a smaller reward. In two recent attempts to explain incentive contrast, Capaldi $(1972,1974)$ has extended his theory to incorporate the relative effects of reinforcement by assigning equal importance to both absolute and relative stimuli. Black (1968) had earlier proposed a modification of Spence's theory which also acknowledged the role that relative stimuli play in the determination of contrast effects.

It is thus proposed that after the following assumptions concerning incentive motivation $(\mathrm{K})$ are made, the reintroduction of $\mathrm{K}$ into Capaldi's theory would make it possible to account, in Hullian terms, for both negative and positive contrast effects, at least under conditions which control for ceiling effects. First, it would be assumed that all incentive shifts affect $K$. Second, $K$ builds up as a function of rewarded trials with magnitude of reward (or some other reinforcement parameter) setting the upper limit to which $\mathrm{K}$ can grow. Third, during shifts in reinforcement conditions, the existing $\mathrm{K}$ not only increases (or decreases) appropriately but receives gradual increments (or decrements) in value proportional to the weighted average of the pre- and postshift reinforcement conditions. This incremental or decremental averaging process approaches zero as a limit. Fourth, K, like V, is presumed to have a high responsivity coefficient. It would probably be necessary but not crucial at this point to reconsider the role of reward magnitude in determining $\mathrm{H}$. However, in line with Capaldi's theory it will be assumed that due to generalization decrement only $70 \%$ of the $\mathrm{H}$ will be available following either an upward or downward shift. It is clear that the latter assumptions provide the bases for predicting both positive and negative contrast as well as accounting for the eventual disappearance of these effects. ${ }^{1}$

\section{REFERENCES}

Amsel, A. The role of frustrative nonreward in noncontinuous reward situations. Psychological Bulletin, 1958, 55, 102-119.

Black, R. W. Shifts in magnitude of reward and contrast effects in instrumental and selective learning: A reinterpretation. Psychological Review, 1968, 75, 114-126.

Bower, G. H. A contrast effect in differential conditioning. Journal of Experimental Psychology, 1961, 62, 196-199.

Capaldi, E. J. A sequential hypothesis of instrumental learning. In K. Spence \& J. T. Spence (Eds.), The psychology of learning and motivation. New York: Academic Press, 1967.

Capaldi, E. J. Successive negative contrast effect. Intertrial interval, type of shift, and four sources of generalization decrement. Journal of Experimental Psychology, 1972, 96, $433-438$.

Capaldi, E. J. Partial reward either following or preceding consistent reward: A case of reinforcement level. Journal of Experimental Psychology, 1974, 102, 954-962.

Dunham, P. J. Contrasted conditions of reinforcement: A selective critique. Psychological Bulletin, 1968, 69, 295-315.

Harris, S. J., Smith, M. G., \& Weinstock, S. Effects of nonreinforcement on subsequent reinforced running behavior. Journal of Experimental Psychology, 1962, 64, 388-392.

Helson, H. Adaptation-level theory: An experimental and systematic approach to behavior. New York: Harper \& Row, 1964.

Leung, C. M., \& Jensen, G. D. Shifts in percentage of reinforcement viewed as changes in incentive. Journal of Experimental Psychology, 1968, 76, 291-296.

Marx, M. Positive contrast in instrumental learning from qualitative shift in incentive. Psychonomic Science, 1969, 16 , 254-255.

Mellgren, R. L. Positive and negative contrast effects using delayed reinforcement. Learning and Motivation, 1972, 3 , 185-193.

Shanab, M. E., \& Biller, J. D. Positive contrast in the runway obtained following a shift in both delay and magnitude of reward. Learning and Motivation, 1972, 3, 179-184.

Shanab, M. E., Birnbaum, D. W., \& Cavallaro, G. Positive contrast obtained in reacquisition following interpolation of nonreinforced or partially reinforced trials. Learning and Motivation, 1974, 5, 258-271.

Shanab, M. E., Sanders, R. \& Premack, D. Positive contrast obtained with delay of reward. Science, 1969, 164, 724-725.

\section{NOTE}

1. The present formulation assumes that excitatory potential (E) is a multiplicative function of habit $(H)$, incentive motivation (K), and such constant factors as drive (D), stimulus-intensity dynamism $(V)$, etc. In mathematical terms, $E=H \times K \times c$, where $c$ stands for the constant factors. Depending on whether an upward or downward shift is ir:olved, the general equation can be rewritten as follows: $E=\bar{H}\left[K+\left(\sqrt{\bar{K}_{S} \times \bar{K}_{I}}\right)^{1 / n}\right] \times c$, for upward shifts, and $\mathrm{E}=\overrightarrow{\mathrm{H}}\left[\mathrm{K}-\left(\sqrt{\mathrm{K}_{\mathrm{S}} \times \mathrm{K}_{\mathrm{L}}}\right)^{1 / n}\right] \times \mathrm{c}$, for downward shifts, where $K_{L}$ and $K_{S}$ stand, respectively, for the $K$ value associated with large and small reward, $n$ refers to number of rewarded trials, and $\overline{\mathrm{H}}$ stands for generalized habit. It is understood that the values of $K$ and $\vec{H}$ in the foregoing equations will change appropriately to correspond to the values associated with the new reward magnitude and number of rewarded trials respectively.

(Received for publication October 29, 1974.) 